Hat sich im Laufe der Zeit die Temperatur des Leitungswassers und der Umgebung so weit verändert, daß der Thermostat das Temperaturgleichgewicht nicht mehr erreichen kann, so muß der Wasserregulierhahn wieder von neuem eingestellt werden, er wird etwas aufgedreht, wenn das Leitungswasser wärmer, etwas zugedreht, wenn es kälter geworden ist.

Die Einteilung am Wasserregulierhahn gestattet, die richtige Lage desselben wieder aufzufinden, die einer bestimmten Temperatur des Leitungswassers entspricht. Die Schwankungen der Badtemperatur betragen nur Bruchteile eines Zehntelgrades, sie sind daher für die meisten Arbeiten belanglos.

Der stündliche Verbrauch an Gas ist ca. 501 , an Wasser, je nach der Temperatur desselben, 15 bis $50 \mathrm{l}$, an Elektrizität 0,046 Kilowatt. Die Betriebskosten betragen danach für die hiesigen Verhältnisse $5^{\mathbf{1}} / \mathbf{2}-6$ Heller pro Stunde.

Der Elektromotor ( $1 / 16$ PS.) ist jedoch so gewählt worden, daß er zum gleichzeitigen Betreiben von anderen Apparaten benutzt werden kann.

Bei Anwendung eines kleineren Elektromotors (1/32 PS.), der zum Betreiben des Thermostatenrührwerkes genügend wäre, sind die Betriebskosten viel kleiner, sie würden für die hiesigen Verhältnisse ca. $3^{1 /} / 2-4$ Heller pro Stunde betragen.

\section{Die schnelle elektroanalytische Bestimmung von Zink.}

\author{
Von Francis C. Frart.
}

\author{
(Eingeg. d. 18./. 1907.)
}

Für die elektroanalytische Bestimmung des Zinks mittels stationärer Elektroden hat man den Gebrauch von den. Salzen verschiedener schwacher Säuren, sowie von Ätzkali und Ätznatron in dem Elektrolyten empfohlen. Letztere scheinen nach den Arbeiten von $S$ pitze r ${ }^{1}$ ) und anderen die brauchbarsten Resultate mit den einfachsten Bedingungen $\mathrm{zu}$ geben.

Mit r o ti ere n d e r A n ode und einer versilberten Platinschale als Kathode bekamen $\mathbf{E} \mathbf{x}$.

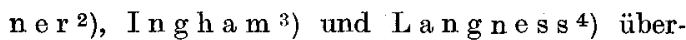
einstimmende Resultate aus Lösungen von $\mathrm{Na}$ triumzinkat unter Zusatz von $2-12 \mathrm{~g} \mathrm{NaOH}$. Exner und Ingh am erzielten brauchbare Niederschläge aus einem Acetatelektrolyten, und I $\mathbf{n g ~ h}$ a $\mathrm{m}$ hat auch ameisensäurehaltige Lösungen untersucht. Medwa $\mathrm{y}^{6}$ ) hat mittels rotierender Kathode das Zink aus dem wohlbekannten Doppeltoxalat bestimmt. Unter Kühlung mit Eis erzielten Pri c e und $\mathrm{J} \mathrm{u} \mathrm{d} \mathrm{g} \mathrm{e}^{6}$ ) brauchbare Resultate aus einer $\mathrm{ZnSO}_{4}$-Lösung unter Zusatz von $\mathrm{Na}_{2} \mathrm{SO}_{4}$.

1) Z. f. Elektrochem. 11, 391 .

2) J. Am. Chem. Soc. 25, 896; Chem. Zentralbl. 1903, II. 1210 .

3) J. Am. Chem. Soc. 26, 1269; Chem. Zentralbl. 1904, II, 1758 .

4) J. Am. Chem. Soc. 29, 459 (1907).

5) Z. anorg. Chem. 42, 114.

6) Chem. News 94, 8 (1906).
Schnellmethoden zur Zinkbestimmung unter Verwendung einer Quecksilberkathode sind auch ausgearbeitet worden, aber ihre Besprechung erübrigt sich hier.

Während vorläufiger Versuche über die Bestimmung des Zinks mit magnetischer Rührung des Elektrolyten wurde bemerkt, daß der Acetatelektrolyt immer einen grob krystallinischen Niederschlag lieferte. Dieser haftete zwar genügend fest an einer Schalenelektrode, doch ließ er sich allzu leicht von der weniger geschützten Oberfläche der Drahtnetzkathode abkratzen. Auch war der Widerstand des Elektrolyten sehr hoch, so daß man oft Spannungen von über 19 Volt brauchen mußte, welche den Elektrolyten bald in lebhaftes Kochen versetzen. Weil Oxalsäure und Ameisensäure ähnliche Störungen hervorrufen, wurde von diesen abgesehen, und der Natriumzinkatelektrolyt ausschließlich für diese Untersuchung benutzt. Letzterer zeigte sich als für Schnellmethoden sehr geeignet; sein Widerstand ist klein, so daß man immer mit 5 oder höchstens 6 Volt auskommt. Die Erfahrung von anderen, daß die $\mathrm{NaOH}-M e n g e$ zwischen 2 und $12 \mathrm{~g}$ variiert werden darf, wurde zum Teil bestätigt, jedoch zeigte sich an den beiden Extremen eine Tendenz zur Schwammbildung, es wurden daher gewöhnlich $7-8 \mathrm{~g}$ gebraucht.

In allen Versuchen, wo der Elektrolyt gerührt wurde, benutzte ich eine zylindrische Kathode aus Nickeldrahtnetz. Nach der Wägung wurde das Zink von der Kathode mit verdünnter $\mathrm{H}_{2} \mathrm{SO}_{4}$ abgelöst; die Nickelmenge, welche sich mit löste, hing von der Säurekonzentration und der Einwirkungszeit ab und schwankte zwischen 0,0 und 10,4 mg. Im Durchschnitt betrug sie ca. I mg pro Bestimmung. Die Höhe der Kathode war immer $5 \mathrm{~cm}$, und ihr Durchmesser wurde so gewählt, daß sie dicht an die Wand des Becherglases paßte.

Die Anode war eine Spirale aus $1 \mathrm{~mm}$ starkem Platindraht; ich merkte, daß sie bald anfing, gelblichbraun zu werden. Nach fünfmaligem Gebrauch wurde sie also gewaschen und in einem Gemisch aus $\mathrm{HNO}_{3}$ und $\mathrm{H}_{2} \mathrm{C}_{2} \mathrm{O}_{4}$ gekocht, um das Bleisuperoxyd, welches ich als die Ursache der Färbung annahm, zu entfernen. Die Farbe blieb unverändert. Die Anode wurde bei $100-110^{\circ}$ getrocknet und gewogen. Nach weiterem Gebrauch in 20 Bestimmunge wurde sie nochmals gewaschen, getrocknet und gewogen und ein Gewichtsverlust von $0,8 \mathrm{mg}$ konstatiert. Ohne weitere Behandlung mit Säuren wurde sie während 46 weiterer Bestimmungen gebraucht und dann nochmals gewaschen, getrocknet und gewogen.

Diesmal wurde ein weiterer Verlust von $0,7 \mathrm{mg}$ gefunden. Die Anode wurde dann mit $\mathrm{HNO}_{3}$ und $\mathrm{H}_{2} \mathrm{C}_{2} \mathrm{O}_{4}$ nochmals gekocht, gewaschen, getrocknet und gewogen. Thre braune Farbe blieb unverändert; eine Gewichtsabnahme von nur $0,2 \mathrm{mg}$ wurde gefunden. Da sich nichts löste, erschien es möglich, daß ein Platinoxyd7) vielleicht die Färbung hervorgerufen hatte. Lag dieses vor, so mußte es bei verhältnismäßig niedriger Temperatur zer-

7) Spätere Anmerkung. Nachdem diese Arbeit abgeschlossen war, fand ich eine Abhandlung von C. $\mathrm{M}$ a $\mathrm{r}$ i e (Compt. r. d. Acad. d. sciences 145, 117 [1907], in der er behauptet, da $B$ dieses ein Peroxyd ist. 
fallen; daher wurde die Spirale einmal schnell durch die Flamme einer Gebläselampe gezogen, aber kaum bis zur Rotglut erhitzt. Trotzdem war sie jetzt vollständig weiß geworden, obwohl ihre Oberfläche, in Gegensatz zu der des oberen Teils des Drahtes, etwas matt aussah. Sie wurde jetzt geglüht, im Exsiccator gekühlt und gewogen. Es zeigte sich ein Verlust von $0,5 \mathrm{mg}$, das Gewicht blieb konstant bei nochmaligem Abspülen mit Wasser und Alkohol und Trocknen, so daß der Verlust nicht auf eine Wasserhaut zurückzuführen ist. Es scheint, daß das Oxyd, welches $S$ e $n$ t e ${ }^{8}{ }^{8}$ nicht aus alkalischen Lösungen gewinnen konnte, hier vorliegt. Wenigstens scheint die Lösungsgeschwindigkeit der Anode so klein zu sein, daß sie für die Analyse nicht in Betracht kommt.

Die Deckgläser und die Wand des Becherglases wurden fünf Minuten vor dem Ende jeder Analyse abgespült; in den Fällen aber, wo der Strom während des zweiten Teils der Elektrolyse klein sein sollte, fand das Abspülen gleich nach der Stromverminderung statt.

Das Volumen des Elektrolyten betrug 100-125 ccm. In den meisten Fällen wurde das Becherglas mit einer Spirale aus $4 \mathrm{~mm}$ starkem Bleirohr umgeben, welche zum Zwecke der Kühlung mit Leitungswasser gespeist war.

Am Ende der Bestimmung wurde die Zelle ohne Stromunterbrechung in der üblichen Weise ausgewaschen. Man läßt den Niederschlag nicht eher an die Luft gelangen, bis fast alles Alkali ausgewaschen ist. Die ersten $200 \mathrm{cem}$ der abgesaugten
Flüssigkeit wurden reserviert zweoks Prüfung ihres Zinkgehalts. Nach Abtropfen des darauf haftenden Waschwassers wurde die Kathode in einen Becher mit Alkohol eingetaucht und dann in einem Trockenschrank bei $100-120^{\circ}$ getrocknet, im Exsiccator gekühlt und gewogen.

Die zuerst abgesaugte Lauge wurde mit $\mathrm{H}_{2} \mathrm{SO}_{4}$ angesäuert und mit $3-5 \mathrm{ccm} \mathrm{K}_{4} \mathrm{Fe}(\mathrm{CN})_{6}(1: 10)$ auf Zink geprüft. Nach Zusatz des Blutlaugensalzes läßt man die Flüssigkeit 10 Minuten stehen, wenn nicht eher eine Trübung stattfindet. Unter diesen Bedingungen gibt $0,2 \mathrm{mg}$ Zink eine deutliche Trübung.

Exner und Ingham erzielten übereinstimmende Resultate bei 5 Amp. und 5-6 Volt in 15-20 Minuten. Scheinbar haben sie das Mittel der Resultate als richtig angenommen und danach den Fehler berechnet. In keinem Falle geben sie an, daß sie die abgesaugte Lauge geprüft hätten, und diese Bemerkung gilt auch für die späteren Arbeiten von $\mathrm{M}$ e d w a y und L a $\mathrm{ng} \mathrm{n}$ es s.

Die Versuche in Tabelle 1 und 2 zeigen, daß man in der Tat übereinstimmende Resultate unter verschiedenen Bedingungen erzielen kann, welche aber alle zu niedrig sind. In fast jedem Falle läßt sich das Zink in der abgesaugten Lauge nachweisen. Läßt man die Prüfung der abgesaugten Flüssigkeit wegfallen, so scheint es, daß die Bestimmung in 35-40 Minuten fertig ist. Die Versuche in Tabelle 2 zeigen, daß mit 4,5 Amp. die Hauptmenge des Metalls schon nach 15 Minuten niedergeschlagen ist, daß aber die letzten Spuren sich sehr schwer von der Lauge trennen lassen.

Tabelle 1 .

Elektrolyse unter magnetischer Rührung`), ohne Kühlung. Nickeldrahtnetzkathode.

\begin{tabular}{|c|c|c|c|c|c|c|c|c|c|}
\hline \multirow{2}{*}{ Nr. } & \multirow{2}{*}{$\begin{array}{l}\text { Lö- } \\
\text { sung }\end{array}$} & \multirow{2}{*}{$\stackrel{Z \mathbf{n}}{\text { gefunden }}$} & \multirow{2}{*}{$\begin{array}{l}\text { Fehler } \\
\text { (mg.) }\end{array}$} & \multirow{2}{*}{ Amp. } & \multirow{2}{*}{ Volt } & \multirow{2}{*}{$\begin{array}{l}\text { Zeit } \\
(\text { Min.) }\end{array}$} & \multicolumn{2}{|c|}{ Temperatur am } & \multirow{2}{*}{$\begin{array}{l}\text { Prüfung der abgesaugten } \\
\text { Flüssigkeit ergab von } \mathrm{Zn}\end{array}$} \\
\hline & & & & & & & Anfang & Schluls & \\
\hline 1 & $\mathrm{~A}$ & 0,1965 & $-1,5$ & 7,4 & - & 33 & kalt & 一 & Spuren \\
\hline 2 & A & 0,1968 & $-1,2$ & 5,9 & 4,3 & 34 & warm & - & , \\
\hline 3 & A & 0,1966 & $-1,4$ & 5,8 & 4,4 & 40 & warm & - & , \\
\hline 4 & B & 0,0912 & $-0,9$ & 4,3 & - & 35 & kalt & $60^{\circ}$ & , \\
\hline 5 & B & 0,0914 & $-0,7$ & 4,5 & - & 50 & kalt & - & , \\
\hline 6 & B & 0,0915 & $-0,6$ & 6,2 & 4,0 & 50 & kalt & $90^{\circ}$ & (verloren) \\
\hline 7 & B & 0,0916 & $-0,5$ & 6,5 & 3,6 & 50 & kalt & $91^{\circ}$ & keins \\
\hline
\end{tabular}

Ta be 11 e 2 .

Lösung C, unter Kühlung und magnetischer Rührung des Elektrolyten. Nickeldrahtnetzkathode. Anfangs Zimmertemperatur.

\begin{tabular}{|c|c|c|c|c|c|c|c|}
\hline 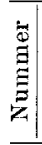 & $\begin{array}{c}\mathrm{Zn} \\
\text { ge- } \\
\text { funden }\end{array}$ & $\begin{array}{l}\text { Fehler } \\
\text { (mg) }\end{array}$ & 皇 & 苛 & 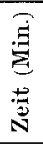 & 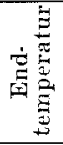 & $\begin{array}{c}\text { Prüfung der } \\
\text { abgesaugten } \\
\text { Flüssigkeit } \\
\text { ergab von } Z n\end{array}$ \\
\hline 1 & 0,1770 & $-15,8$ & 4,4 & 4,4 & 10 & $30^{\circ}$ & viel \\
\hline 2 & 0,1920 & $-0,8$ & 4,5 & 4,6 & 15 & $37^{\circ}$ & Spuren \\
\hline 3 & 0,1916 & $-\quad 1,2$ & 4,4 & 4,6 & 25 & $36^{\circ}$ & . \\
\hline 4 & 0,1922 & $-0,6$ & 4,5 & 4,2 & 31 & $44^{\circ}$ & ", \\
\hline $\mathbf{5}$ & 0,1925 & $-0,3$ & 5,3 & 4,3 & 45 & $50^{\circ}$ & \\
\hline 6 & 0,1932 & $+\quad 0,4$ & 4,5 & 4,1 & 51. & $39^{\circ}$ & geringe Spuren \\
\hline
\end{tabular}

8) Trans. Faraday Soc. 2, 142 (1906); nach J. chem. soe, 92, II, 68.

9) Unter Gebrauch meines, in einer früheren Mitteilung (diese Z. 20, Heft 44, 1894 [1907]) beschriebenen Apparates für diesen Zweck.
Im Gegensatz hierzu zeigen die Versuche in Tabelle5 (S. 2250), daß man bei richtigen Bedingungen alles Zink in 35 Minuten ausfällen kann.

Da es wahrscheinlich ersehien, daß das Zink unter den Bedingungen von $\mathrm{E} \times \mathrm{n}$ e $\mathrm{r}$ und $\mathrm{I} \mathrm{n} \mathrm{g}$ $\mathrm{h}$ a $\mathrm{m}$ nicht vollständig niedergeschlagen war, wurde eine Reihenfolge von qualitativen Versuchen mit rotierender Anode und einer Nickelschale als Kathode durchgeführt (Tabelle 3 ).

Die Resultate zeigen, daß die Bestimmung nach der Methode dieser Autoren (nur geändert im Gebrauch einer Nickelschale statt einer versilberten Platinschale) in der Tat nach fast einer Stunde noch nicht beendet war, und daß in dieser Beziehung das Verhalten des Elektrolyten mit rotierender Anode dasselbe ist wie mit magnetischer Rührung, was auch zu erwarten war. Ubrigens ist das Unterlassen der Prüfung der abgesaugten Flüssigkeit ein Fehler, welcher nicht scharf genug verurteilt werden kann, 
welcher jedoch in vielen Untersuchungen elektroanalytischer Methoden zu finden ist.

Es war zu erwarten, daß die Temperatur des Elektrolyten einen großen Einfluß auf die Geschwindigkeit der Fällung üben würde, da das Zink sich in Natronlauge löst, und seine Lösungstension mit der Temperatur steigt. In den Arbeiten von Exn e r, In $\mathrm{g} h$ a $m$ und $L$ a $n g$ es $s$ wurde die Temperatur nicht beobachtet, and ihr Einfluß

$$
\text { T a b e } 11 \text { e } 3 \text {. }
$$

Qualitative Versuche mit rotierender Anode und Nickelschale. Anfangs Zimmertemperatur.

Zinkmenge $0,2 \mathrm{~g}$.

\begin{tabular}{c|c|c|c|c|c}
\hline Nr. & Amp. & Volt & $\begin{array}{c}\text { Zeit } \\
\text { (Min.) }\end{array}$ & $\begin{array}{c}\text { End- } \\
\text { tem- } \\
\text { peratur }\end{array}$ & $\begin{array}{c}\text { Prüfung der abge- } \\
\text { saugten Flüssigkeit } \\
\text { ergab von Zn }\end{array}$ \\
\hline \hline 1 & 4,8 & 4,8 & 20 & $40^{\circ}$ & viel \\
2 & 5 & - & 17 & - & viel \\
3 & 5,3 & 4,5 & 30 & - & Spuren \\
4 & 5,3 & 5 & 35 & $53^{\circ}$ & Spuren \\
5 & 4,9 & 4,8 & 47 & - & geringe Spuren \\
6 & 4,9 & 4,8 & 57 & $52^{\circ}$ & geringe Spuren
\end{tabular}

überhaupt nicht besprochen. $A \mathrm{~m} \mathrm{~b}$ e $\left.\mathrm{r} \mathrm{g}^{10}\right)$, der obne Rotation mit sehr starker Kalilauge arbeitete, fand, daß bei $60^{\circ}$ das Metall oft schwammig ausfiel. Bei $40^{\circ}$ war der Niederschlag gut, aber es erwies sich nicht als vorteilhaft, die Lösung auf dieser Temperatur zu halten. Daher empfiehlt er, den Elektrolyten zuerst auf $60-70^{\circ} \mathrm{zu}$ erhitzen und dann die Flamme wegzunehmen, so daß er sich von selbst auf beinahe Zimmertemperatur abkühlt.
S pit z er ${ }^{11}$ ) hat den Effekt der Temperatur nicht besonders untersucht, er begnügt sich mit der Tatsache, daß die Erhitzung des Elektrolyten unnötig ist, und daß die Fällung bei Zimmertemperatur sehr gut erfolgt. Gr e n e ${ }^{12}$ ) im Gegenteil empfiehlt eine Temperatur von $50^{\circ}$ für die Bestimmung.

Ich finde in Ubereinstimmung mit $\mathrm{A} \mathrm{m}$ b e $\mathrm{r} \mathrm{g}$, daß der Niederschlag, den man aus einem heißen Elektrolyten erhält, sehr oft schwammig oder wenigstens dunkel gefärbt ist; ich finde auch, daß es bei hohen Temperaturen sehr schwer ist, die letzten Spuren des Zinks zu fällen. Bei den höheren Stromdichten, welche für schnelle Fällung nötig sind, erscheint eine andere Schwierigkeit, nämlich die, daß sich das Zink bei solchen scheinbar leichter in der Lauge wieder löst als bei niedriger Stromdichte. Daher habe ich es für nötig gefunden, zur Fällung von $0,2 \mathrm{~g}$ Zink einen Strom von ca. 4,5 Amp. während der ersten 15 Minuten zu gebrauchen und dann die Elektrolyse mit 1-2 Amp. 15--20 Minuten fortzusetzen. Während des zweiten Teils der Elektrolyse fällt auch die Temperatur, weil die $\mathrm{J}$ o u l e sche Wärmetönung viel kleiner ist. Die Versuche in Tabelle 2 zeigen, daß es wenig Zweck hat, den starken Strom mehr als 15 Minuten zu gebrauchen, weil dann bis auf etwa $1 \mathrm{mg}$ schon alles gefällt ist, und bei längerer Elektrolyse die Temperatur fortwährend steigt.

Wenn aber am Ende der ersten 15 Minuten der Strom bis auf $1-2$ Amp. vermindert und die Elektrolyse fortgesetzt wird, schlägt sich. das ganze Zink nieder, wie in Versuchen 1, 3 und 5, Tabelle 4 (ohne Kühlung) und in Tabelle 5 (mit Kühlung).

Tabelle 4.

Lösung A unter magnetischer Rührung aber ohne Kühlung des Elektrolyten. Nickeldrahtnetzkathode. Verminderter Strom während des zweiten Teils der Elektrolyse. Anfangs Zimmertemperatur.

\begin{tabular}{c|c|c|c|c|c|c|c|c|c|c}
\hline \hline \multirow{2}{*}{ Nr. } & \multirow{2}{*}{$\begin{array}{c}\text { Zn } \\
\text { gefunden }\end{array}$} & $\begin{array}{c}\text { Fehler } \\
\text { (mg) }\end{array}$ & \multicolumn{2}{|c|}{ Amp. } & \multicolumn{2}{|c|}{ Volt } & \multicolumn{3}{|c|}{ Zeit } & \multicolumn{2}{c}{ Puüfung der abgesaugten } \\
Flüssigkeit ergab von Zn
\end{tabular}

Versuche 2, 4 und 6, Tabelle 4, zeigen, daß die Stromverminderung allein nicht immer zufriedenstellende Resultate gibt; während mit Kühlung und Stromverminderung (Tabelle 5) die Methode sehr zuverlässig ist. Der Strom durch das Solenoid wurde immer auf 4,5-5 Amp. gehalten, so daß die Rührung lebendig blieb.

Wenn weniger als $0,1 \mathrm{~g}$ Zink in der Lösung ist, scheint die Stromverminderung nicht unbedingt nötig, wie aus Tabelle $6 \mathrm{zu}$ ersehen ist.

Es wäre aber doch sicherer, auch hier mit den Bedingungen von Tabelle 5 zu arbeiten.

Um zu zeigen, daß die Schnellmethode Resultate gibt, welche mit denen der gewöhnlichen $\mathrm{Me}$ thode übereinstimmen, wurden fünf Analysen von Lösung A ohne Rührung in einer Nickelschale

10) Berl. Berichte 36, 2489 (1903). durchgeführt. Der Elektrolyt enthielt ca. $8 \mathrm{~g}$ $\mathrm{NaOH}$, der Strom war 0,8-1 Amp., und kein Zink ließ sich in der abgesaugten Lauge nachweisen. Die gefundenen Zinkmengen waren 0,$1973 ; 0,1986$; 0,$1978 ; 0,1985$ und $0,1990 \mathrm{~g}$; also im Mittel $0,1982 \mathrm{~g}$. Das Mittel der Schnellanalysen von Lösung A, wo die Fällung vollständig war (Nr. 2, 4 und 6, Tab. 4) ist $0,1983 \mathrm{~g}$. Für die anderen zwei Lösungen $\mathrm{B}$ und $\mathrm{C}$ nahm ich als den Zinkgehalt das Mittel aller der Bestimmungen an, bei denen das ganze Zink gefällt war (außer Nr. 5 und 8, Tabelle 5) und berechnete den Fehler der einzelnen Bestimmungen danach. Die angenommenen Werte waren : Lösung $\mathrm{B}=0,0921 \mathrm{~g}$, Lösung $\mathrm{C}=0,1928 \mathrm{~g}$.

Die Niederschläge waren meistens von fast

11) Loc. cit.

12) Chem.-Ztg. 1905, 803. 
Tabelle 5.

Lösung C, unter Kühlung und magnetischer Rührung des Elektrolyten. Nickeldrahtnetzkathode. Während des zweiten Teils der Elektrolyse wurde der Strom vermindert. Anfangs Zimmertemperatur.

\begin{tabular}{|c|c|c|c|c|c|c|c|c|c|c|c|}
\hline \multirow{2}{*}{$\mathrm{Nr}$. } & \multirow{2}{*}{$\begin{array}{c}\mathrm{Zn} \\
\text { gefunden }\end{array}$} & \multirow{2}{*}{$\begin{array}{l}\text { Fehler } \\
(\mathrm{mg})\end{array}$} & \multicolumn{2}{|c|}{$A$} & \multicolumn{2}{|c|}{ Volt } & \multicolumn{3}{|c|}{ Zeit (Min.) } & \multirow{2}{*}{$\begin{array}{c}\text { SchlnB- } \\
\text { tem- } \\
\text { peratur }\end{array}$} & \multirow{2}{*}{$\begin{array}{l}\text { Prifung der abge- } \\
\text { saugten Flüssigkeit } \\
\text { +rgab ron } \mathrm{Zn}\end{array}$} \\
\hline & & & $\mathbf{a}$ & b & $\mathbf{a}$ & $\mathrm{b}$ & $\mathrm{a}$ & $\mathrm{b}$ & $a+b$ & & \\
\hline 1 & 0,1926 & $-0,2$ & 4,5 & 1,1 & 4,6 & 3,2 & 17 & 23 & 40 & $23^{\circ}$ & keins \\
\hline 2 & 0,1930 & $+0,2$ & 4,5 & 1,2 & 4,6 & 3,1 & 20 & 15 & 35 & $31^{\circ}$ & keins \\
\hline 3 & 0,1934 & $+0,6$ & 4,5 & 1,2 & 一 & - & 17 & 17 & 34 & 一 & geringe Spuren \\
\hline 4 & 0,1926 & $-0,2$ & 4,7 & 1,7 & 4,5 & 3,3 & 18 & 18 & 36 & $28^{\circ}$ & keins \\
\hline 5 & 0,1944 & $+1,6$ & 4,7 & 1,7 & 4,2 & 3,6 & 15 & 20 & 35 & $25^{\circ}$ & keins 13) \\
\hline 6 & 0,1930 & $+0,2$ & 4,4 & 1,7 & 4,3 & 3,3 & 15 & 17 & 32 & $35^{\circ}$ & keins \\
\hline 7 & 0,1926 & $-0,2$ & 4,4 & 1,7 & 4,3 & 3,5 & 15 & 17 & 32 & $26^{\circ}$ & geringe Spuren \\
\hline 8 & 0,1944 & $+1,6$ & 4,5 & 0,9 & 4,5 & 3,0 & 25 & 17 & 42 & $22^{\circ}$ & keins 13 ) \\
\hline 9 & 0,1930 & $+0,2$ & 4,5 & 0,9 & 4,3 & - & 15 & 17 & 32 & $28^{\circ}$ & geringe Spuren \\
\hline
\end{tabular}

weißer Farbe und metallischem Glanz. Ferner wurde gefunden, daB die Beschaffenheit des Niederschlages sehr von der der Kathodenoberfläche abhing; auf der einen Kathode, die sehr lange mit Säuren behandelt worden war, war der Niederschlag

Ta belle 6 .

Lösung B unter Kühlung und magnetischer Rüh-, rung des Elektrolyten. Nickeldrahtnetzkathode. Anfangs Zimmertemperatur.

\begin{tabular}{|c|c|c|c|c|c|c|c|}
\hline Nr. & $\begin{array}{c}\mathrm{Zn} \\
\text { ge- } \\
\text { funden }\end{array}$ & $\begin{array}{c}\text { Fehler } \\
\text { (mg) }\end{array}$ & $\dot{\bar{\Xi}}$ & $\stackrel{\overrightarrow{0}}{0}$ & 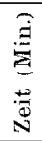 & 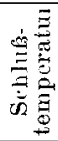 & $\begin{array}{c}\text { Prüfung der } \\
\text { abgesaugten } \\
\text { Flüssigkeit } \\
\text { ergab von } \mathrm{Zn}\end{array}$ \\
\hline 1 & 0,0902 & $-1,9$ & 5,3 & 4,6 & 30 & $52^{\circ}$ & Spuren \\
\hline 2 & 0,0908 & $-1,3$ & 4,3 & 4,4 & 25 & $41^{\circ}$ & \\
\hline 3 & 0,0920 & $-0,1$ & 4,3 & 4,1 & 30 & - & keins \\
\hline 4 & 0,0924 & $+0,3$ & 4,5 & 4,5 & 30 & - & \\
\hline 5 & 0,0920 & $-0,1$ & 4,5 & - & 30 & - & \\
\hline 6 & 0,0924 & $+0,3$ & 4,5 & - & 32 & $47^{\circ}$ & \\
\hline 7 & 0,0910 & $-1,1$ & 4,5 & - & 35 & $45^{\circ}$ & Spuren \\
\hline 8 & 0,0912 & $-0,9$ & 4,4 & 4,6 & 32 & $38^{\circ}$ & keins \\
\hline
\end{tabular}

immer von etwas dunklerer Farbe und zeigt eine Tendenz sich zu oxydieren, wie bei Versuchen 5 und 8 , Tabelle 5, bei welchen diese Kathode gebraucht wurde.

\section{$\mathrm{Zusammentassung.}$}

i. Mit elektromagnetischer Rührung des Elektrolyten läßt sich $0,1 \mathrm{~g}$ Zink auf einer Nickeldrahtnetzkathode durch $4,5 \mathrm{Amp}$. in 30 Minuten vollständig niederschlagen. Man braucht ca. $8 \mathrm{~g} \mathrm{NaOH}$ in dem Elektrolyten und kühlt denselben.

2. Für Mengen von Zink bis $0,2 \mathrm{~g}$ gebraucht man 4-5 Amp. während 15 Minuten und dann ca. 1,5 Amp. während weiterer 20 Minuten. Die anderen Bedingungen wie in 1.

3. Die Prüfung der abgesaugten Flüssigkeit ist notwendig.

4. Es ist höchst unwahrscheinlich, daß vollständige Fällung von $0,2 \mathrm{~g}$ Zink in 15-20 Minuten mit 5 Amp. bewirkt wird, wie Ex $\mathrm{n}$ er und In $\mathrm{g}$ $\mathrm{h}$ a $\mathrm{m}$ angeben. Nach den obigen Versuchen scheint es auch unwahrscheinlich, daB die übereinstimmen-

13) Auf einer rauhen Kathode niedergeschlagen; dunkel gefärbt und wahrscheinlich oxydiert. den Resultate von Mis s Lang ness, wonach das Zink in 3 Vinuten mittels 10-13 Amp. gefällt war, vollständige Fällungen darstellen.

B e r 1 i n, 17. Juli 1907.

Elektrochemisches Laboratorium der Kgl. Technischen Hochschule.

\section{Erwiderung auf den Artikel von Prof. Schulze=Pillot über Ver $=$ suche an D. T. S.=Exhaustoren.}

Von Prof. Gr. Lindner in Karlsruhe i. B.

(Eingeg. d. 2./11. 1907.)

Prof. $\mathrm{Sehulze}-\mathrm{Pill}$ ot hat in Heft 2 des Jahrganges 1907 dieser Zeitschrift einen Aufsatz veröffentlicht, in dem er meinen früheren Bericht über Steinzeugexhaustoren angreift. Seine Vorwürfe sind durchweg ungerechtfertigt und bedürfen einer sachlichen Richtigstellung.

$\mathrm{Schulze-Pillot}$ fand bei Versuchen an den Fixhaustoren der Deutschen Ton- und Steinzeugwerke in Charlottenburg größere Windmengen, als ich an einem Exhaustor dieser Firma und in gleicher Weise an Exhaustoren der Deutschen Steinzeugfabrik in Friedrichsfeld i. B. gemessen habe. Die beiderseitigen $\mathrm{M}$ e $\beta \mathrm{v}$ e $\mathrm{r} f \mathrm{a} h \mathrm{r}$ en sind grundsätzlich so verschieden, daß ein unmittelbarer Vergleich der Zahlenwerte oder Kurven gar nicht statthaft ist. Das hat $S$ c h u l z e Pill ot nicht beachtet.

Ich ließ den Exhaustor in einen an den Blasehals unmittelbar angesetzten großen Kasten mit seitJicher Ausströmung blasen. Er benutzte einen mittels konischen Stutzens an den Blasehals anschlieBenden Kessel mit Ausströmung am hinteren Ende. Das Geheimnis des Unterschiedes liegt hauptsächlich in dem $\mathrm{Kegelstutzen}$, der als sogen. Diffusor in bekannter Weise wirkt: Die Strömungsgeschwindigkeit im Blasehals verzögert sich bei der allmählichen Ausbreitung des Stromes in dem konischen Übergang und setzt sich (mit einigen Prozenten Verlust) in meßbare Druckhöhe um. Hierbei wird die Energie der Strömung aus. 